\title{
Wie ist die Prognose bei zerebralen Cavernomen?
}

\begin{abstract}
Zerebrale Cavernome treten bei einem von etwa 600 neurologisch gesunden Menschen auf. Ihre Bedeutung wurde erst mit Einführung der Kernspintomografie erkannt, da sie meist im CT nicht sichtbar sind. Sie können im Prinzip drei Komplikationen hervorrufen: zerebrale Blutung, fokal-neurologische Ausfälle und epileptische Anfälle. Zuverlässige Zahlen zum Spontanverlauf fehlten bislang.
\end{abstract}

— Eine prospektive populationsbezogene Studie aus Schottland erfasste alle Fälle von Cavernomen zwischen 1999 und 2003. Primärer Endpunkt war die Kombination aus intrazerebralen Blutungen oder fokal-neurologischen Ausfällen mit Ausnahme von epileptischen Anfällen in Zusammenhang mit Cavernomen.

Die Erstdiagnose eines Cavernoms wurde bei 139 Patienten gestellt. Davon hatten 24 multiple Cavernome. Bei 47\% erfolgte die Diagnose zufällig, bei $25 \%$ im Rahmen der Abklärung epileptischer Anfälle, bei $12 \%$ nach intrakranieller
Blutung und bei $15 \%$ bestanden fokalneurologische Ausfälle. 63\% der Cavernome waren lobär, $14 \%$ im Hirnstamm, $13 \%$ im Kleinhirn und 6\% in den Basalganglien lokalisiert. Das 5-Jahres-Risiko für eine erste Blutung $(2,4 \%)$ war deutlich geringer als das Risiko einer erneuten Blutung (29,5\%). Das 5-Jahres-Risiko des primären Endpunktes betrug 9,3\%, für ein erneutes Auftreten nach fünf Jahren $42,4 \%$. Die meisten Ereignisse traten im ersten Jahr nach Diagnosestellung auf.

\section{Kommentar}

Diese Studie ist die erste populationsbezogene Untersuchung zur Häufigkeit und den klinischen Konsequenzen von Cavernomen. Wichtig ist die Information, dass Cavernome offenbar relativ harmlos sind, solange sie keine Symptome erzeugen. Wenn es aber zu einer ersten Blutung gekommen ist, nimmt das Risiko erneuter Blutungskomplikationen deutlich zu. Daher muss die Entscheidung, ob ein Cavernom operativ versorgt wird, relativ rasch getroffen werden.

H.-C. DIENER =

\section{- R. A. Salman et al.}

Untreated clinical course of cerebral cavernous

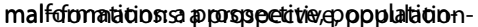
based cohort study. Lancet Neurol 11 (2012) 217-224

\section{Frühe Menopause erhöht das Osteoporoserisiko und die Mortalität}

\begin{abstract}
1977 wurden 390 Frauen im Alter von 48 Jahren für die Malmö Perimenopausal Study rekrutiert. Untersucht werden sollte, wie sich der frühzeitige Beginn der Wechseljahre langfristig auswirkt.
\end{abstract}

— Die Frauen wurden in zwei Kategorien unterteilt: Frauen, deren Menopause begonnen hatte, bevor sie 47 Jahre alt

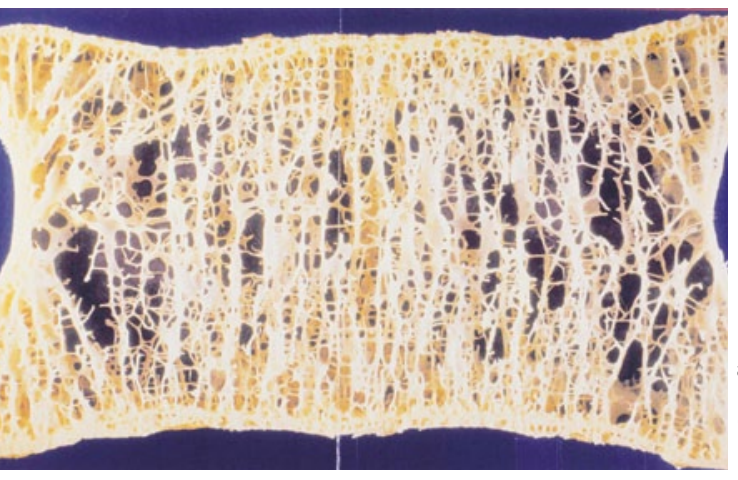

waren und Frauen, deren Menopause im Alter von 47 Jahren oder später eintrat. Im Alter von 77 Jahren wurde bei den verbleibenden Probandinnen erneut eine Knochendichtemessung durchgeführt. Zwischenzeitlich verstorben waren 92 Frauen, 100 der 298 verbliebenen Frauen waren entweder weggezogen und daher nicht mehr greifbar oder lehnten eine weitere Teilnahme ab, sodass schließlich 198 Probandinnen weiter an der Studie teilnahmen.

$56 \%$ der Frauen mit einer frühzeitigen Menopause hatten Osteoporose im Vergleich zu 30\% der Frauen mit der späteren Menopause. Die Mortalität lag in der Gruppe mit der frühen Menopau-

Bei früher Menopause fast $60 \%$ Osteoporose. se bei $52,4 \%$ (Vergleichsgruppe $35,2 \%$ ). Die Häufigkeit für Knochenbrüche lag bei $44,3 \%$ vs. $30,7 \%$.

\section{- O. Svejme et al.}

(Dept of Orthopaedics, Skane University Hospital, S-20502, Sweden; E-mail: ola.svejme@med. lu.se ) Early menopause and risk of osteoporosis, fracture and mortality : a 34-year prospective observational study in 390 women. Published online on 25 April 2012 in BJOG 2012:; DOI: 10.1111/j.1471-0528.2012.03324.x.

\section{Kommentar}

Eine frühzeitige Menopause ist ein signifikanter Risikofaktor für Osteoporose, Knochenbrüche und verfrühte Sterblichkeit. Noch nicht untersucht wurde, ob sich das Ernährungsverhalten, der Alkoholkonsum, die Medikamenteneinnahme und der Tabakkonsum in den beiden Gruppen unterschied und ob hierin möglicherweise der Grund für die unterschiedlichen Osteoporose- und Mortalitätsraten lag. 